\title{
MODIFIED NORWOOD PROCEDURE WITH A HIGH-FLOW CARDIOPULMONARY BYPASS STRATEGY RESULTS IN LOW MORTALITY WITHOUT LATE ARCH OBSTRUCTION
}

Nancy C. Poirier, FRCSC ${ }^{a}$

Jonathan J. Drummond-Webb, MB, BCh, $\mathrm{FCS}(\mathrm{SA})^{\mathrm{a}}$

Kunikazu Hisamochi, MD, $\mathrm{PhD}^{\mathrm{a}}$

Michiaki Imamura, $\mathrm{MD}, \mathrm{PhD}^{\mathrm{a}}$

A. Marc Harrison, MD $^{\mathrm{b}}$

Roger B. B. Mee, MB, ChB, FRACS ${ }^{a}$
Objective: The results of our modification of the stage I Norwood procedure, in which we use only autologous tissue to reconstruct the aortic arch, were reviewed. A high-flow, low-pressure cardiopulmonary bypass protocol (with phenoxybenzamine), before and after a period of deep hypothermic circulatory arrest, was used.

Methods: Between 1993 and 1999, 59 patients, aged 1 to 353 days (median 4 days) and weighing 1.7 to $6.8 \mathrm{~kg}$ (median $3.2 \mathrm{~kg}$ ), underwent a modified Norwood procedure. The ascending aortic diameter ranged from 1.5 to $8 \mathrm{~mm}$ (median $3 \mathrm{~mm}$ ). The modified Blalock-Taussig shunt was $3 \mathrm{~mm}$ in 21 patients $(36 \%)$ and $3.5 \mathrm{~mm}$ or larger in 38 patients $(64 \%)$.

Results: Deep hypothermic circulatory arrest and cardiopulmonary bypass times ranged from 15 to 64 minutes (median 37 minutes) and 44 to 144 minutes (median 88 minutes), respectively. Early postoperative survival was $83 \%$. By univariate analysis, early mortality was associated with an ascending aortic diameter of $2.5 \mathrm{~mm}$ or less $(P=.01)$. Weight, circulatory arrest and bypass times, diagnosis (hypoplastic left heart syndrome vs variant), shunt size, and date of the procedure did not affect survival. For a median followup period of 37 months (range 4-63 months), 42 (61\%) patients underwent bidirectional cavopulmonary shunts, 10 (17\%) had Fontan operations, and 1 patient underwent transplantation after a bidirectional cavopulmonary shunt. Eight patients subsequently died, for a 1-year actuarial survival of $72 \%(95 \%$ confidence interval: $60 \%-84 \%$ ). Neoaortic arch obstruction was corrected in 3 patients $(5 \%)$.

Conclusions: At intermediate-term follow-up, our modification of the Norwood procedure together with our perioperative strategies has resulted in acceptable outcomes with a low incidence of neoaortic arch obstruction. Patients with a small ascending aortic diameter have emerged as a high-risk group, but a recent technical modification may improve the outlook for these patients. (J Thorac Cardiovasc Surg 2000;120:875-84)
From the Center for Pediatric and Congenital Heart Disease ${ }^{\mathrm{a}}$ and the Department of Pediatric Critical Care, ${ }^{b}$ Cleveland Clinic Foundation, Cleveland, Ohio.

Read at the Eightieth Annual Meeting of The American Association for Thoracic Surgery, Toronto, Ontario, Canada, April 30-May 3, 2000.

Received for publication May 4, 2000; accepted for publication June 12, 2000.

Address for reprints: Roger B. B. Mee, Chairman and Surgical Director, Center for Pediatric and Congenital Heart Disease, Cleveland Clinic Foundation, M/41, 9500 Euclid Ave, Cleveland, OH 44195 (E-mail: meer@ccf.org).

Copyright (C) 2000 by The American Association for Thoracic Surgery

0022-5223/2000 $\$ 12.00+0 \quad \mathbf{1 2 / 6 / 1 0 9 5 4 0}$

doi: $10.1067 / \mathrm{mtc} .2000 .109540$
$T$ he prospects for patients with hypoplastic left heart syndrome (HLHS) and similar anatomic variants were dramatically altered with the introduction of a staged palliative approach as described by Norwood and colleagues. ${ }^{1}$ The first stage involves the creation of an unobstructed communication between the systemic right ventricle through a reconstructed aorta and arch with a controlled source of pulmonary blood flow through a systemic-pulmonary shunt. ${ }^{1-3}$ As the child outgrows the arterial shunt and the pulmonary vascular resistance decreases, a bidirectional cavopulmonary shunt (BCPS) is performed and the Fontan operation, or a variant thereof, is completed as a final procedure. This 




Fig 1. The modified Norwood procedure: The ascending aorta, arch, descending aorta, and brachiocephalic arteries were completely mobilized. The MPA was transected obliquely, proximal to the right and left pulmonary arteries.

staged approach is an attractive alternative to primary cardiac transplantation.

The Norwood procedure, however, remains challenging, with a relatively high early and late mortality. The "classic" Norwood procedure, in which the ascending aorta and arch are augmented with homograft material, has a reported incidence of arch obstruction of $14 \%$ to $33 \%$ due to lack of growth, calcification, and degeneration. ${ }^{4-6}$ Branch pulmonary artery obstruction from a redundant reconstructed arch has also been reported. ${ }^{7}$

Our modification of the Norwood procedure consists of augmenting the arch by means of a combined anastomosis of the proximal main pulmonary artery (MPA) to the arch and descending aorta, without the use of foreign material. ${ }^{8}$ In addition to the technical modification, the perioperative strategy has evolved and features high-flow, low-pressure perfusion made possible with the use of phenoxybenzamine, judicious management of fluid balance, a low threshold for peritoneal dialysis, and ventilation customized to the physiology of the patient.

The early and intermediate results of our therapeutic strategy for HLHS and its variants are presented here.

\section{Patients and methods}

Patients. Between August 1993 and July 1999, 59 consecutive infants ( 37 boys and 22 girls) with HLHS or a variant of HLHS underwent a modified Norwood procedure. The median age at operation was 4 days (range 1-353 days, mean $19 \pm$ 59 days), including 4 patients older than 2 months $(74,79$,
Table I. HLHS variants: Anatomic features

\begin{tabular}{lc}
\hline Diagnosis & No. of patients $(n=12)$ \\
\hline HLHS and VSD & 1 \\
Unbalanced CAVC & 2 \\
Complex DORV & 4 \\
TA and TGA & 2 \\
DILV and TGA & 3 \\
LA isomerism & 2 \\
\hline
\end{tabular}

$\overline{H L H S \text {, Hypoplastic left heart syndrome; } C A V C \text {, complete atrioventricular }}$ canal; $D O R V$, double-outlet right ventricle; $T A$, tricuspid atresia; $T G A$, transposition of the great arteries; DILV, double-inlet left ventricle; $L A$, left atrial.

Table II. Associated lesions $(n=12)$

\begin{tabular}{lc}
\hline Lesion & No. \\
\hline Persistent LSVC & 5 \\
Aberrant RSCA & 4 \\
ALCARPA & 1 \\
PAPVR & 1 \\
TAPVR & 1 \\
\hline
\end{tabular}

LSCV, Left superior vena cava; RSCA, right subclavian artery; ALCAPA, anomalous left coronary artery from right pulmonary artery; $P A P V D$, partial anomalous pulmonary venous drainage; TAPVD, total anomalous pulmonary venous drainage.

288, and 353 days). Five infants were premature, the youngest born after 31 weeks of gestation. The median weight was $3.2 \mathrm{~kg}$ (range 1.7-6.8 kg, mean $3.3 \pm 0.81 \mathrm{~kg}$ ).

Forty-seven patients $(80 \%)$ had HLHS, defined as mitral and aortic stenosis or atresia (or both) in the presence of normally related great vessels, a hypoplastic left ventricle, and an intact ventricular septum. Patients who did not conform to these criteria but who had a duct-dependent parallel circulation, with a nonseptatable heart and left ventricular outflow tract obstruction, were considered to have variants of HLHS. The anatomic features of the HLHS variants are listed in Table I.

An aberrant right subclavian artery was encountered in 4 patients. The coronary sinus was unroofed in 2 of the 5 patients with a persistent left superior vena cava. An interrupted aortic arch was present in 4 patients. Associated lesions are listed in Table II.

The diameter of the ascending aorta was measured by echocardiogram before the operation and verified during the operation. The diameters of the ascending aorta ranged from 1.5 to $8 \mathrm{~mm}$ (median $3 \mathrm{~mm}$; mean $3.5 \pm 1.6 \mathrm{~mm}$ ). Twentyfour patients had an ascending aorta measuring $2.5 \mathrm{~mm}$ or smaller. Antegrade flow in the ascending aorta was absent in 27 patients (46\%). The mitral valve was atretic in 19 patients $(32 \%)$.

Tricuspid or atrioventricular valve regurgitation (TR) was documented with color flow Doppler echocardiography. TR was described as trace or mild in 45 patients $(76 \%)$, moderate in $11(19 \%)$, and severe in $1(2 \%)$. Only 1 patient had a preoperative balloon atrial septostomy at another institution. 


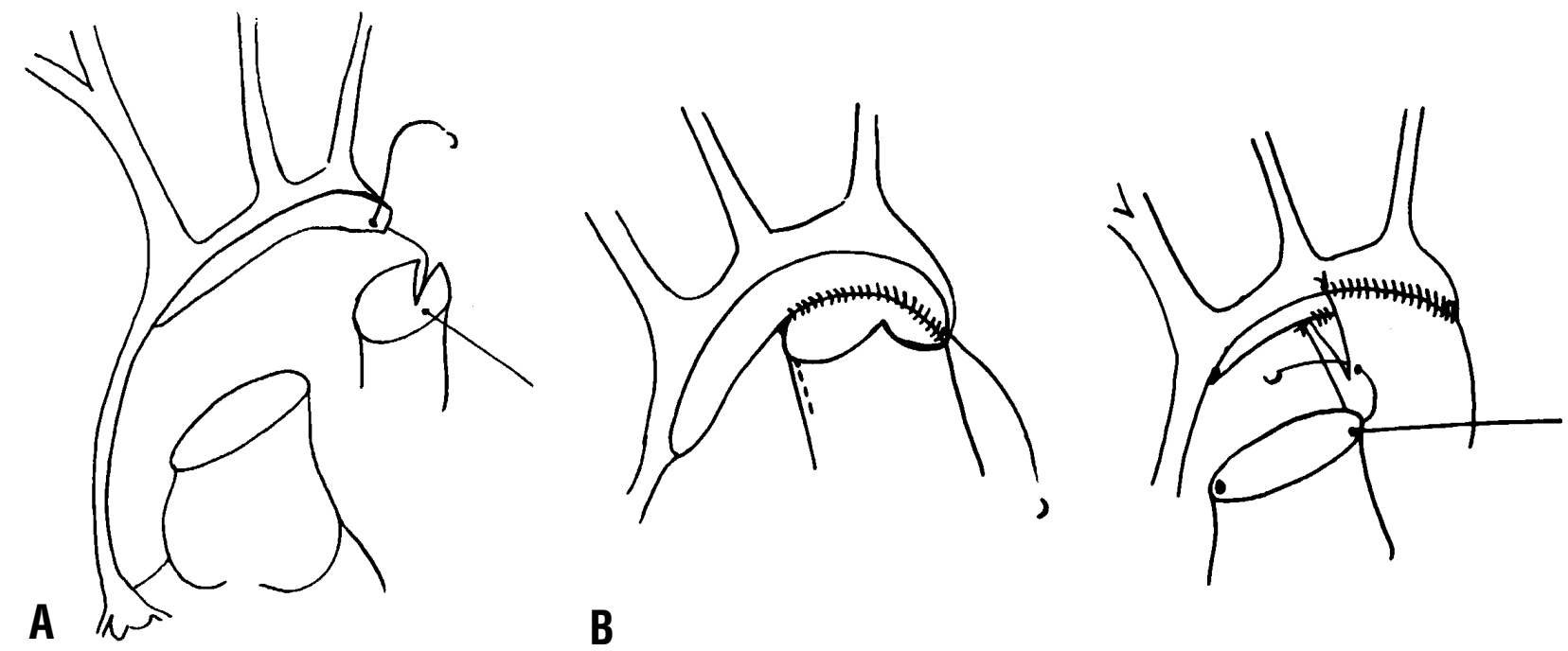

Fig 2. The modified Norwood procedure (continued): A, Once all of the ductal tissue was resected, the lesser curvature of the arch was opened from the left subclavian artery to the ascending aorta. B, The arch was reconstructed by anastomosis of the descending aorta to the aortic isthmus and distal arch. The proximal MPA was then sutured to a combination of ascending aorta, proximal arch, and descending aorta.

Preoperatively, 47 patients $(80 \%)$ were in stable condition with good peripheral perfusion and little or no acidosis. The other 12 patients $(20 \%)$ had severe hemodynamic instability with systemic malperfusion and end-organ failure. Necrotizing enterocolitis developed preoperatively in 1 patient, and surgery was postponed while he was medically managed. Fifty-one patients $(86 \%)$ received mechanical ventilation before the operation, intubated either electively or due to hemodynamic instability. Alprostadil (prostaglandin $\mathrm{E}_{1}$ ) was administered preoperatively in all but the 4 patients who presented after 2 months of age. Preoperative inotropic support was required in 26 patients (44\%). All patients were subjected to ultrasound evaluation of the head and abdomen before undergoing the modified Norwood procedure.

Perfusion protocol. The perfusion protocol was standardized. Methylprednisolone $(20 \mathrm{mg} / \mathrm{kg})$ was administered before cardiopulmonary bypass (CPB). Fresh, heparinized whole blood was used in the CPB prime. Phenoxybenzamine $(0.25 \mathrm{mg} / \mathrm{kg})$ facilitated vasodilation necessary to allow high perfusion flow (greater than $150 \mathrm{~mL} \cdot \mathrm{kg}^{-1} \cdot \mathrm{min}^{-1}$ ) before and after the period of circulatory arrest. Perfusion pressures were maintained at 30 to $35 \mathrm{~mm} \mathrm{Hg}$ and body temperatures were cooled to $18^{\circ} \mathrm{C}$ (nasopharyngeal).

Operative technique. A median sternotomy and subtotal thymectomy were performed, exposing the pericardium, which was harvested for the reconstruction of the pulmonary artery confluence. CPB was established with the arterial cannula placed in the ductus arteriosus (double cannulation for an interrupted aortic arch) and a single right atrial venous cannula. The ascending aorta, arch, descending aorta, and brachiocephalic arteries were completely mobilized. The main pulmonary artery was transected obliquely, proximal to the origins of the right and left pulmonary arteries (Fig 1).
The distal MPA was closed with a fresh, autologous pericardial patch. A modified Blalock-Taussig shunt was fashioned with a polytetrafluoroethylene (PTFE) tube graft and the proximal anastomosis on the distal innominate artery was completed during cooling. A 3-mm PTFE tube graft was used in 20 patients (34\%), a $3.5-\mathrm{mm}$ graft in $34(58 \%)$, and a classic Blalock-Taussig shunt was performed in a $1.7-\mathrm{kg}$ child. Among the 4 older patients, 3 received a 4-mm PTFE tube graft and the fourth received a 5-mm PTFE tube graft.

Deep hypothermic circulatory arrest (DHCA) was instituted at $18^{\circ} \mathrm{C}$, and the cannulas were removed after the heart was arrested with a single dose of crystalloid cardioplegic solution delivered through the arterial cannula. All ductal tissue was resected, and the lesser curvature of the arch was opened from the left subclavian artery to the ascending aorta (Fig 2). In 43 patients (73\%) this incision was extended at the level of the innominate artery in the patients in whom the diameter of the ascending aorta was larger or to the midascending aorta when the ascending aorta was smaller. The arch was reconstructed by anastomosis of the descending aorta to the aortic isthmus and distal arch. The proximal MPA was then anastomosed to a combination of ascending aorta, proximal arch, and descending aorta.

Concerns with the anastomosis of the MPA to a small ascending aorta have resulted in a series of modifications of this technique (Figs 3 to 5). In 16 patients, the ascending aorta was transected and shortened. It was then reimplanted onto the innominate artery in 7 patients, the posterior MPA in 3 , and the right anterior sinus of the MPA in 6 patients.

A radical atrial septectomy was then performed through the right atrial cannulation site. CPB flow was restarted and rewarming was begun, during which time the distal anastomosis of the modified Blalock-Taussig shunt was completed. 


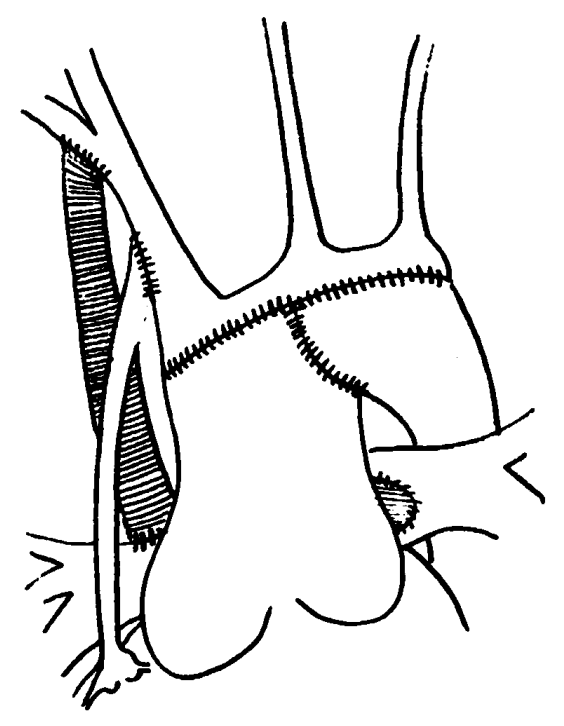

Fig 3. The modified Norwood procedure - the first modification: In 7 patients, the ascending aorta was transected, shortened, and reimplanted onto the proximal innominate artery. There were 3 late deaths at 1.5, 2, and 3 months postoperatively, possibly due to coronary steal from the adjacent modified Blalock-Taussig shunt.

An intracardiac monitoring line was placed in the left atrium. After administration of protamine, a peritoneal dialysis catheter was inserted. Before the patient was weaned from $\mathrm{CPB}$, dopamine $\left(5 \mu \mathrm{g} \cdot \mathrm{kg}^{-1} \cdot \mathrm{min}^{-1}\right)$ and nitroglycerin $(5 \mu \mathrm{g}$ $\left.\cdot \mathrm{kg}^{-1} \cdot \mathrm{min}^{-1}\right)$ were started. Norepinephrine $\left(0.1-0.4 \mu \mathrm{g} \cdot \mathrm{kg}^{-1}\right.$ - $\min ^{-1}$ ) was added if systemic vasodilation due to the phenoxybenzamine was excessive. The chest was closed in all but 9 patients (15\%), in whom there were intraoperative hemodynamic changes. The sternum was subsequently closed at a median of 2.5 days after the operation (range 2-4 days).

Postoperative management. Patients were kept sedated and paralyzed during the initial 24 hours. Ventilatory adjustments were used to balance pulmonary and systemic blood flows. A pressure-regulated volume-controlled mode of ventilation was used. Initial settings included a rate of 20 to 25 breaths $/ \mathrm{min}$, a positive end-expiratory pressure of 4 to $6 \mathrm{~cm} \mathrm{H}_{2} \mathrm{O}$, and a preset tidal volume of $12 \mathrm{~mL} / \mathrm{kg}$. Ventilatory goals were a normal $\mathrm{pH}$ and an arterial carbon dioxide level between 35 and $45 \mathrm{~mm}$ $\mathrm{Hg}$. The inspired oxygen fraction was adjusted to maintain transcutaneous oxygen saturations between $70 \%$ and $80 \%$ and an arterial oxygen level of 35 to $45 \mathrm{~mm} \mathrm{Hg}$. If and when the oxygen saturation was consistently above $80 \%$ with the patient breathing room air, the preset tidal volume was then decreased to maintain a mild respiratory acidosis, and the arterial carbon dioxide level was kept between 45 and $55 \mathrm{~mm} \mathrm{Hg}$. Hypoxic admixtures were not used in any of our patients. In the presence of high pulmonary vascular resistance and desaturation, nitroglycerin infusion was then combined with alprostadil or inhaled nitric oxide (or both).

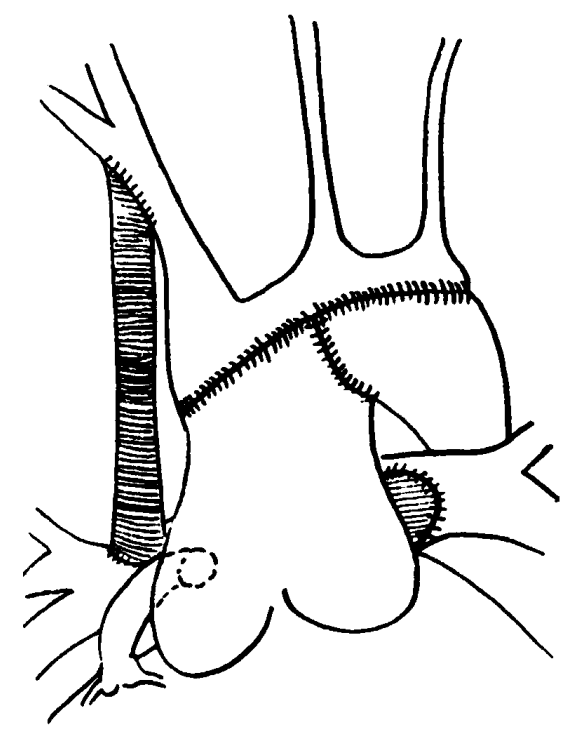

Fig 4. The modified Norwood procedure-the second modification: The ascending aorta was shortened and implanted onto the posterior face of the main pulmonary artery, which would preserve the lie of the ascending aorta. This was quickly abandoned after early deaths in 2 of the 3 patients who underwent this procedure. Myocardial ischemia by compression of the ascending aorta was believed to be the culprit.

Peritoneal dialysis administered by a previously described technique $^{9}$ was instituted for decreased urine output or increased serum potassium levels.

All patients received anticoagulation in the form of heparin (10 units $\cdot \mathrm{kg}^{-1} \cdot \mathrm{h}^{-1}$ ) for the initial 48 hours, and long-term acetylsalicylic acid ( $5 \mathrm{mg} / \mathrm{kg}$ every other day) was begun on postoperative day 1. Feeding was begun gradually, first in the form of continuous feeds, followed by gavage feeding through a nasogastric tube, and finally oral feeding was begun as tolerated. Strict attention to fluid balance and homeostasis was maintained in the early postoperative period.

BCPS and Fontan operations. Before BCPS and Fontan operations, an echocardiogram and cardiac catheterization were obtained. Both procedures were performed through a median sternotomy with $\mathrm{CPB}$, without circulatory arrest. The pulmonary arteries were augmented with fresh autologous pericardium in the presence of stenoses.

The Fontan operation was performed at approximately 3 to 5 years of age with the development of activity-limiting exercise intolerance or cyanosis. A lateral tunnel Fontan was created by means of a PTFE patch with a 3- to 4-mm fenestration.

Statistical methods. Clinical and follow-up data were retrieved from medical records. Statistical analysis was obtained by means of Statistica 4.0 software (StatSoft, Inc, Tulsa, Okla). Early postoperative death was defined as occurring within 30 days of the modified Norwood procedure or during the same hospitalization. The data are reported as a median, range, and/or mean \pm standard deviation. 
The significance of continuous variables on early mortality was determined by means of the Mann-Whitney rank sum test. Categoric variables were analyzed with the use of a $\chi^{2}$ test or a Fisher exact 2-tailed test if one of the numerators of a proportion was equal to or less than 5 events.

The actuarial survival was estimated by a Kaplan-Meier method and $95 \%$ confidence limits (95\% CI) were stated. The attrition rate was centered around the first 5 months after the modified Norwood procedure. The effect of the variables analyzed for early postoperative mortality was also calculated for the intermediate postoperative mortality. Univariate analysis of continuous variables was therefore performed by means of the Cox proportional hazard regression model, and categoric variables underwent a log-rank test.

\section{Results}

DHCA and CPB times ranged from 15 to 64 minutes (median 37 minutes, mean $39 \pm 9$ minutes) and 44 to 144 minutes (median 88 minutes, mean $96 \pm 24$ minutes), respectively.

The early postoperative survival was $83 \%$ (49/59). An autopsy was obtained in 5 of the 10 patients who died. In 2 patients a transmural infarct of the right and left ventricles was observed. Ischemic myocardial changes were observed in a patient who died of a postoperative respiratory syncytial virus infection and tamponade. A clotted shunt was found in 1 patient and a severely dysplastic tricuspid valve in another.

Early reinterventions were necessary in 9 patients (15\%) with 2 early deaths. Indications for reoperation included pericardial effusion in 2 patients $(3 \%)$, tamponade in $3(5 \%)$ after removal of intracardiac lines or pacing wires, and shunt thrombosis in $2(3 \%)$. Revision of the neoaortic arch was required within 24 hours of the initial operation in 2 patients (3\%). Extracorporeal membrane oxygenation was instituted after postoperative cardiac arrest in 3 patients, all of whom were successfully weaned and discharged from the hospital. One of the 3 patients died suddenly 3 months after the operation, another is developmentally delayed, and the third patient is doing well, with a slight decrease in fine motor skills, and is soon to be evaluated for a Fontan procedure.

Postoperative complications included sepsis in 13 (22\%) patients (including mediastinitis in 2 patients [3\%]), supraventricular tachyarrhythmia in 4 (7\%), chylothorax lasting more than 1 week in 1 patient $(2 \%)$, and medically treated necrotizing enterocolitis in 1 patient $(2 \%)$. Four patients had seizures during the postoperative period, including the 3 patients who had a cardiac arrest, were resuscitated, and were supported temporarily with extracorporeal membrane oxygenation. Diaphragmatic palsy was identified in 2 patients,

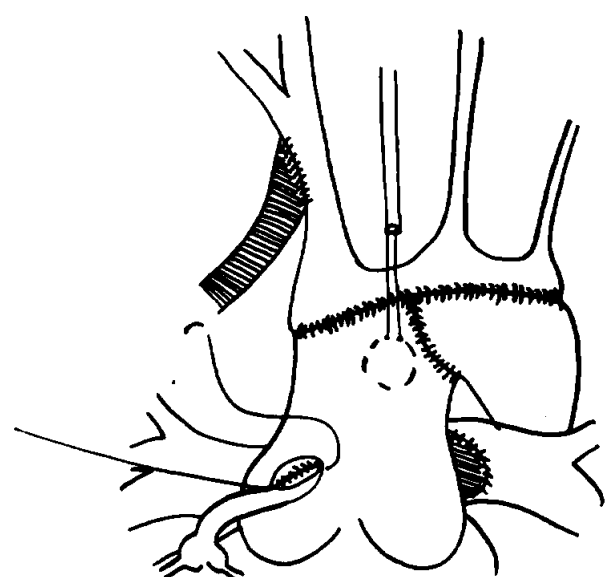

Fig 5. The modified Norwood procedure-the third modification: The ascending aorta was anastomosed onto the anterior rightward sinus of the MPA. Between August 1993 and July 1999, 6 patients underwent this last modification, which was performed with a shortened ascending aorta. One early death and no late deaths were recorded. From July 1999 to March 2000, 7 other patients have had a full-length ascending aorta implanted onto the anterior MPA with 1 early death.

resolving in 1 , and 2 other patients had recurrent laryngeal nerve paralysis, with 1 patient requiring a tracheostomy for upper airway obstruction.

The median hospital stay was 13 days (range 7-112 days, mean $19 \pm 17$ days). The median stay in the critical care unit was 7 days (range 3-99 days, mean $12 \pm$ 15 days), and duration of mechanical ventilation was a median of 5 days (range 1-28 days, mean $6.7 \pm 5.8$ days).

Variables evaluated as potential risk factors for early mortality are listed in Tables III and IV. The size of the ascending aorta $(P=.05)$ and the absence of antegrade flow $(P=.004)$ through the aortic valve have a significant impact on postoperative mortality. Of the 24 patients with a small ascending aorta diameter $(\leq 2.5$ $\mathrm{mm}), 8$ or $33 \%$ died. In contrast, only 2 of the 35 patients $(5.7 \%)$ with an ascending aorta diameter larger than $2.5 \mathrm{~mm}$ died $(P=.01)$. The 1 - and 4 -year actuarial survivals for patients with a small ascending aorta are 50\% (95\% CI: 30\%-70\%) at both time points. Patients with an ascending aorta more than $2.5 \mathrm{~mm}$ in diameter had an actuarial survival at 1 and 4 years of 88\% (95\% CI: $77 \%-99 \%$ ) and $80 \%$ (95\% CI: $62 \%-$ 98\%), respectively ( $P=.006$; Fig 6).

During the initial experience, the ascending aorta was left in situ regardless of the size of the ascending aorta. The early mortality in 13 patients with a small ascending aorta with this technique was $38 \%$. Since 
Table III. Univariate predictors of early and intermediate postoperative mortality: Continuous variables

\begin{tabular}{lcccc}
\hline & & & \multicolumn{2}{c}{ P values } \\
\cline { 4 - 5 } Mortality variable & Median & Range & Early mortality & Intermediate mortality \\
\hline Age (d) & 4.0 & $1-353$ & .05 & .01 \\
Ascending aorta diameter (mm) & 3.0 & $1.5-8.0$ & .02 & .006 \\
Weight (kg) & 3.2 & $1.7-6.8$ & .89 & .46 \\
Circulatory arrest time (min) & 37 & $15-64$ & .36 & .13 \\
CPB (min) & 88 & $56-144$ & .43 & .49 \\
\hline
\end{tabular}

$\overline{C P B}$, Cardiopulmonary bypass time.

Table IV. Univariate predictors of early and intermediate postoperative mortality: Categoric variables

\begin{tabular}{|c|c|c|c|c|c|}
\hline \multirow[b]{2}{*}{ Variable } & \multirow[b]{2}{*}{$n$} & \multicolumn{2}{|c|}{ Early mortality } & \multicolumn{2}{|c|}{ Intermediate mortality } \\
\hline & & $\%$ & $\mathrm{P}$ value & $\%$ & $\mathrm{P}$ value \\
\hline \multicolumn{6}{|l|}{ Age } \\
\hline$<3 \mathrm{~d}$ & 9 & 44 & .03 & 56 & .02 \\
\hline$\geq 3 \mathrm{~d}$ & 50 & 12 & & 26 & \\
\hline \multicolumn{6}{|l|}{ Age } \\
\hline$<5 \mathrm{~d}$ & 27 & 22 & .49 & 41 & .07 \\
\hline$\geq 5 \mathrm{~d}$ & 32 & 12 & & 22 & \\
\hline \multicolumn{6}{|l|}{ Sex } \\
\hline Male & 37 & 19 & .73 & 32 & .36 \\
\hline Female & 22 & 14 & & 27 & \\
\hline \multicolumn{6}{|l|}{ Weight } \\
\hline$<3 \mathrm{~kg}$ & 20 & 15 & 1.00 & 35 & .34 \\
\hline$\geq 3 \mathrm{~kg}$ & 39 & 18 & & 28 & \\
\hline \multicolumn{6}{|l|}{ Ascending aorta diameter } \\
\hline$\leq 2.5 \mathrm{~mm}$ & 24 & 33 & .01 & 50 & .006 \\
\hline$>2.5 \mathrm{~mm}$ & 35 & 5.7 & & 17 & \\
\hline Aortic atresia & 27 & 33 & .004 & 52 & .0001 \\
\hline Aortic stenosis & 32 & 3.1 & & 12 & \\
\hline Mitral atresia & 19 & 32 & .06 & 58 & .004 \\
\hline Mitral stenosis & 40 & 10 & & 18 & \\
\hline HLHS & 47 & 21 & .10 & 36 & .11 \\
\hline HLHS variant & 12 & 0 & & 8 & \\
\hline \multicolumn{6}{|l|}{ Preop TR } \\
\hline$\leq$ Mild & 45 & 11 & .05 & 24 & .05 \\
\hline >Mild & 14 & 36 & & 50 & \\
\hline \multicolumn{6}{|l|}{ Shunt } \\
\hline $3 \mathrm{~mm}$ & 20 & 15 & 1.0 & 40 & .31 \\
\hline$\geq 3.5 \mathrm{~mm}$ & 38 & 18 & & 26 & \\
\hline Preop end-organ failure & 12 & 8.3 & .67 & 25 & .18 \\
\hline \multicolumn{6}{|l|}{ Era } \\
\hline $1993-1996$ & 29 & 10 & .29 & 31 & .27 \\
\hline $1997-1999$ & 30 & 23 & & 30 & \\
\hline
\end{tabular}

HLHS, Hypoplastic left heart syndrome; Preop TR, perioperative tricuspid and atrioventricular valve regurgitation.

then, 3 modifications of the technique have been performed in 16 patients, aimed at reducing the mortality in this high-risk group. Results of individual reimplantation techniques are shown in Figs 3 to 5. The ascending aorta was shortened for these modifications. The first modification with reimplantation onto the innominate artery was associated with 3 late deaths $(n=7)$ at $1.5,2$, and 3 months after the modified Norwood pro- cedure. The hypothesis was coronary steal from the adjacent modified Blalock-Taussig shunt. The second modification involved implantation of the ascending aorta onto the posterior face of the MPA, which would preserve the lie of the ascending aorta. This was quickly abandoned after 2 early deaths $(n=3)$. Myocardial ischemia by compression of the ascending aorta was believed to be the culprit. The last modification con- 


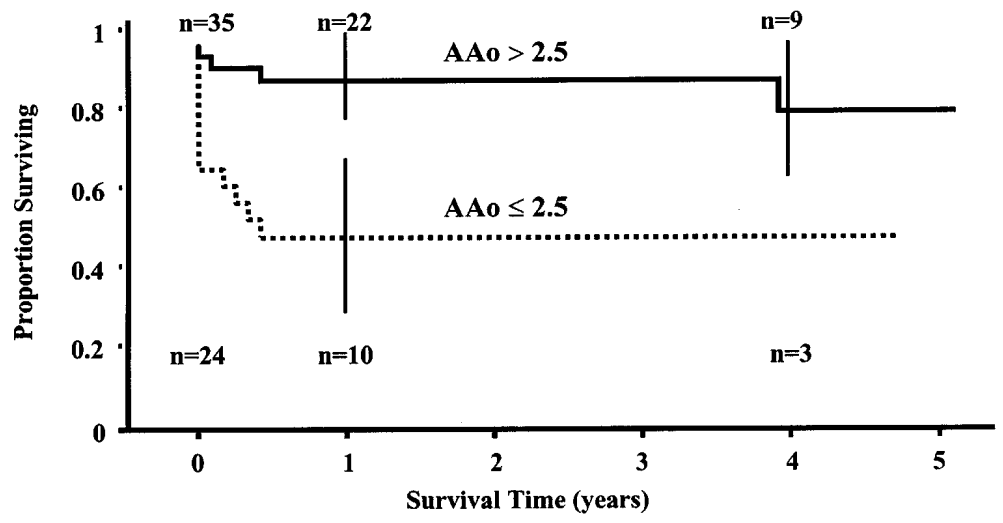

Fig 6. Actuarial survival after the modified Norwood procedure according to ascending aorta size. Ninety-five percent confidence limits are stated. $n$, Number of patients; AAo, ascending aorta diameter.

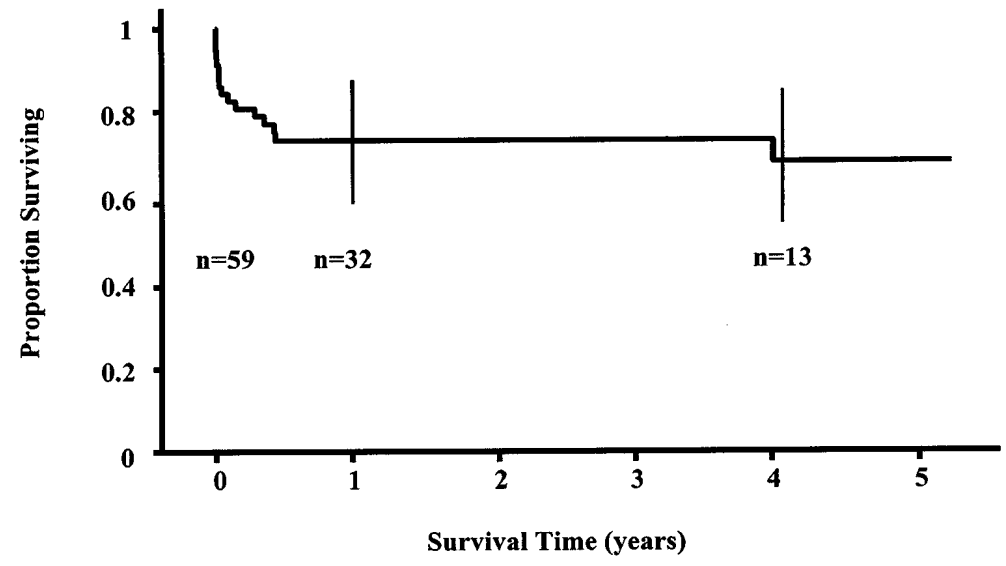

Fig 7. Overall actuarial survival after the modified Norwood procedure. Ninety-five percent confidence limits are stated; $n$, number of patients.

sisted of the implantation of the ascending aorta onto the rightward and anterior sinus of the MPA. Among the 6 patients subjected to this last modification, there has been 1 early death and no late mortality. From July 1999 to March 2000, there have been 7 other patients in whom the full-length ascending aorta has been anastomosed to the anterior MPA. A single patient with documented myofibrillar myopathy has died.

Age at operation was found to affect postoperative mortality $(P=.05)$. Nine patients underwent surgery at less than 3 days of age, and of these 4 died after the operation $(P=.03)$. All early postoperative deaths were in patients aged less than 7 days at operation. Preoperative TR (moderate and severe) is also predictive of postoperative death $(P=.05)$. Two other predictors of mortality beyond the early postoperative period were TR of greater than a mild degree and younger age.
The use of a 3.0-mm modified Blalock-Taussig shunt had no effect on the overall intermediate-term postoperative mortality. Interestingly, preoperative shock and end-organ failure were not significant predictors of early mortality $(P=.67)$. The size of the modified Blalock-Taussig shunt, anatomic diagnosis (variant vs nonvariant), weight, DHCA and CPB times, as well as the era (before and after 1996), were not associated with early mortality (Tables III and IV). There were no early deaths in the 4 patients with an aberrant right subclavian artery who had the modified Blalock-Taussig shunt placed onto the right carotid artery.

During a median follow-up period of 37 months (range 4-66 months), there were 8 deaths, 6 of which were during the first year of life, resulting in 1- and 4year actuarial survivals estimated at $72 \%$ (95\% CI: $60 \%-84 \%$ ) and $68 \%$ (95\% CI: $74 \%-82 \%)$, respectively 


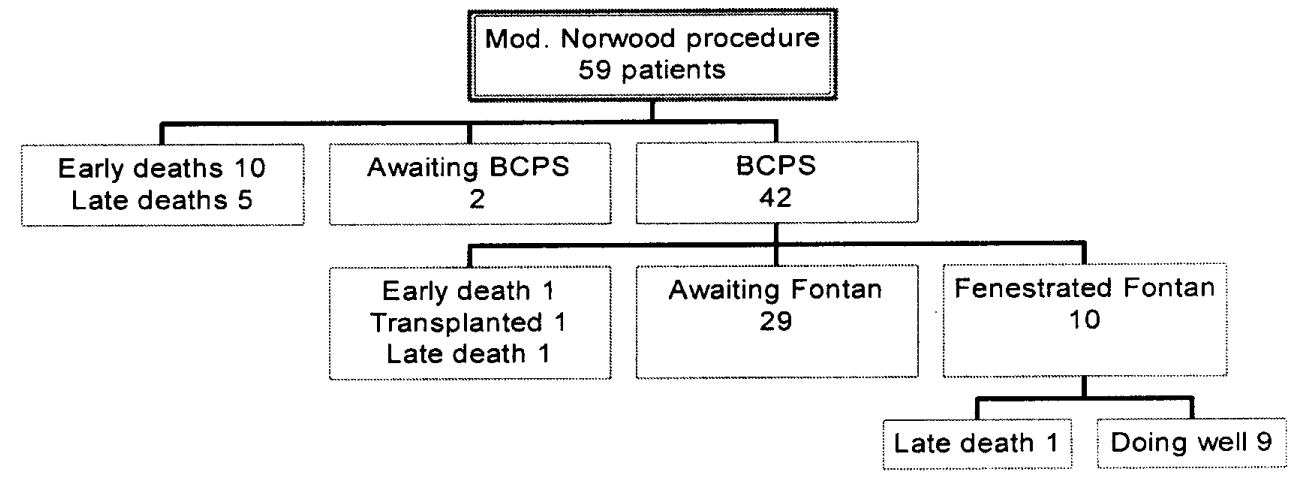

Fig 8. Intermediate follow-up of the modified Norwood procedure. BCPS, Bidirectional cavopulmonary shunt.

(Fig 7). Postmortem examinations were not obtained in these patients. Five deaths occurred before BCPS (Fig 8). Two patients were clinically well and died suddenly, 1 had severe TR and was scheduled for tricuspid valve repair and BCPS, and 1 child died of respiratory complications associated with a documented respiratory syncytial virus. One child aged 3 months died 3 days after balloon dilatation of an ascending aorta/neoaortic suture line stenosis. Two other patients underwent successful balloon dilatation for a $15-$ and $30-\mathrm{mm} \mathrm{Hg}$ gradient at the level of the anastomosis between the MPA and descending aorta. There has been no restenosis 2.5 and 3 years later.

Forty-two patients underwent BCPS at a median age of 5 months (range 3-20 months, mean $5.7 \pm 27$ months). One patient died early of complications from mediastinitis and renal failure. The 1 late death after BCPS was due to accidental head trauma a few days before a scheduled Fontan operation. Cardiac transplantation was performed 2 years after BCPS in 1 child who was unsuitable for a Fontan operation due to severe right ventricular failure.

Ten patients have undergone a fenestrated Fontan procedure at a median age of 4.1 years (range 2.5-5.1 years). The median aortic crossclamp time was $39 \mathrm{~min}$ utes (range 23-51 minutes) and the median CPB time was 82 minutes (range 63-132 minutes). One patient with progressive right ventricular failure died suddenly 3 years after the Fontan operation.

Postoperative echocardiographic data were available in all survivors. The last evaluation was performed at a median of 18 months (range 4-62 months, mean $26 \pm$ 21 months) after the modified Norwood procedure. TR was mild, trace, or absent in 33 children (80\%), moderate in $6(14.6 \%)$, and severe in 1 patient (2\%). Tricuspid annuloplasties have been performed in 7 of
59 patients (12\%), 2 at the modified Norwood procedure, 3 at the BCPS, and 2 at the Fontan operation. No operative deaths occurred in this group of patients and only 1 patient has TR necessitating re-repair at a subsequent staged procedure. Only 1 patient underwent balloon dilation of the left pulmonary artery 2 years after BCPS and underwent a successful Fontan procedure 3 months later. Pulmonary artery patch reconstruction was performed at BCPS or the Fontan operation if the patency of the branch pulmonary arteries was in doubt.

\section{Comment}

Staged reconstruction for HLHS and HLHS variants has resulted in variable outcomes, with reported 5-year actuarial survivals of $20 \%$ to $69 \% .^{6,10-14}$ Most deaths occur in the first year of life, particularly before the BCPS. Since the first descriptions of the Norwood procedure as part of a staged reconstruction protocol, a number of technical modifications have been developed in an attempt to improve outcomes. Advances in surgical technique, small-body perfusion technology, and perioperative management by a multidisciplinary team have resulted in improved survival for this difficult group of patients.

The modified Norwood procedure was possible in all patients without the use of patch augmentation even in the presence of an interrupted aortic arch. A tensionfree reconstruction is possible by extensively mobilizing the descending aorta, arch, and brachiocephalic arteries. The longitudinal split of the medial descending aorta and the use of as much MPA length as possible are technical aspects that further reduce tension on the anastomosis.

At intermediate follow-up, our modification of the Norwood technique achieves results comparable with 
those reported for the standard Norwood operation. ${ }^{10-14}$ Early postoperative survival of the 36 patients with an ascending aorta larger than $2.5 \mathrm{~mm}$ is $94.3 \%$. The $1-$ and 4-year actuarial survivals of $88 \%$ and $80 \%$ are certainly acceptable. However, early on in this series it became evident that a small ascending aorta $(\leq 2.5 \mathrm{~mm})$ was a risk factor with this technique. The high operative mortality associated with a small ascending aorta is not unique to our series and has been observed by other authors using both a patch augmentation to reconstruct the $\operatorname{arch}^{13,14}$ and the modified Norwood technique. ${ }^{6}$

A number of modifications of the aortic reimplantation technique were attempted before we accepted the present approach. This method of reimplantation uses the full length of the small ascending aorta anastomosed end to side into the anterior and rightward aspect of the proximal MPA at the level of the sinotubular junction. The early postoperative mortality in this high-risk group appears to have been substantially reduced, but the numbers are small, follow-up is short, and further evaluation is necessary.

Advantages of the modified Norwood procedure include a low incidence of neoaortic/arch obstruction, avoidance of foreign material, a low incidence of left pulmonary artery compression, and shorter DHCA times. ${ }^{10,11}$ These factors are also essential to satisfactory intermediate and long-term results, providing conditions favorable for a successful BCPS and subsequent Fontan candidacy.

Anatomic features would appear by association to influence outcomes. A small ascending aorta invariably accompanies aortic and mitral atresia. The presence of substantial TR before the modified Norwood procedure may be indicative of ventricular dysfunction, possibly due to myocardial ischemia. In our series, the presence of an aberrant right subclavian artery and modified Blalock-Taussig shunt originating from the right carotid artery was not associated with an increased operative mortality, as reported by Ishino and associates, ${ }^{6}$ using a similar modified Norwood technique. Furthermore, we did not find the diagnosis of an HLHS variant to be associated with a lower operative mortality, as reported by Daebritz and colleagues. ${ }^{11}$

The increased number of early postoperative deaths seen in patients aged less than 7 days is worrisome. This group of patients was in hemodynamically stable condition before the operation, with good systemic perfusion. We suspect that the labile pulmonary vascular circuit, which is normally seen in the first week of life, may have led us to use a larger modified BlalockTaussig shunt, making postoperative management difficult and translating into an unfavorable outcome. A larger cohort of patients would allow for a meaningful multivariate analysis to determine the true impact of age on the perioperative mortality.

The small number of patients in our study precludes statistical analysis of the relative contributions of the anatomic variants, technical details, and perioperative management to the operative mortality. The long-term neurologic outcome of these patients is of concern and presently the subject of ongoing studies.

In conclusion, at intermediate-term follow-up, the combination of our modification of the Norwood procedure together with meticulous perioperative management has resulted in acceptable early and intermediate survival. Patients with a small ascending aorta have emerged as a high-risk group, but a recent technical modification may improve outcomes in this group of patients.

\section{REFERENCES}

1. Norwood WI, Lang P, Hanson DD. Physiologic repair of aortic atresia-hypoplastic left heart syndrome. N Engl J Med 1983;308:23-6.

2. Norwood WI, Lang P, Castaneda AR, Campbell DN. Experience with operations for hypoplastic left heart syndrome. J Thorac Cardiovasc Surg 1981;82:511-9.

3. Norwood WI, Kirklin JK, Sanders SP. Hypoplastic left heart syndrome: experience with palliative surgery. Am J Cardiol 1980;45:87-91.

4. Starnes VA, Griggin ML, Pitlick PT, et al. Current approach to hypoplastic left heart syndrome: Palliation, transplantation, or both? J Thorac Cardiovasc Surg 1992;64:189-95.

5. Bartram U, Grunenfelder J, Van Praagh R. Causes of death after the modified Norwood procedure: a study of 122 postmortem cases. Ann Thorac Surg 1997;64:1795-802.

6. Ishino K, Stumper O, De Giovanni JJV, et al. The modified Norwood procedure for hypoplastic left heart syndrome: early to intermediate results of 120 patients with particular reference to aortic arch repair. J Thorac Cardiovasc Surg 1999;117:920-30.

7. Bove EL, Lloyd TR. Staged reconstruction for hypoplastic left heart syndrome. Ann Surg 1996;224:387-95.

8. Frasier CD, Mee RBB. Modified Norwood procedure for hypoplastic left heart syndrome. Ann Thorac Surg 1995;60:S5469.

9. Mee RBB. The arterial switch operation. In: Stark J, de Leval M, editors. Surgery for congenital heart defects. 2nd ed. Philadelphia: WB Saunders; 1994. p. 483-500.

10. Bove EL. Surgical treatment of hypoplastic left heart syndrome. Jpn J Thorac Cardiovasc Surg 1999;47:47-56.

11. Daebritz SH, Nollert GDA, Zurakowski D, et al. Results of Norwood stage I operation: comparison of hypoplastic left heart syndrome with other malformations. J Thorac Cardiovasc Surg 2000;119:358-67.

12. Jacobs ML, Blackstone EH, Bailey LL. Intermediate survival in neonates with aortic atresia: a multi-institutional study. The Congenital Heart Surgeons Society. J Thorac Cardiovasc Surg 1998;116:417-31.

13. Forbes JM, Cook N, Roth SJ, et al. Ten-year institutional experi- 
ence with palliative surgery for hypoplastic left heart syndrome: risk factors related to stage I mortality. Circulation 1985;92(Suppl):II-262-6.

14. Kern JH, Hayes CJ, Michler RE, Gersony WM, Quagebeur JM. Survival and risk factor analysis for the Norwood procedure for hypoplastic left heart syndrome. Am J Cardiol 1997;80:170-4.

\section{Discussion}

Dr Pedro J. del Nido (Boston, Mass). You have succinctly identified two of the key features of the standard Norwood operation that remain problematic - the management of the diminutive aorta and the use of nonviable tissue as material for aortic augmentation.

Your results are very similar to the results from our series of 194 patients that Dr Daebritz presented last year at this meeting. The overall survival at 1 year is about $87 \%$ in the most recent patient group. However, there were differences with respect to risk factors, and I would be interested in your comments on those differences.

First, we did not identify ascending aortic size as a risk factor, although our current technique for the diminutive ascending aorta is very similar to the one that you described. Is the fact that you identified the size of the ascending aorta as a risk factor simply a reflection of the so-called learning curve because you were trying different techniques? Do you think this will remain a risk factor now that you are using the reimplantation technique? Do you think this is an ongoing risk factor?

Second, we have been concerned about the use of a direct tissue-to-tissue connection between the MPA trunk or the neoaortic root and the transverse arch and descending aorta. That concern stems in part from the potential for left pulmonary artery compression because of the tension generated. I noted that balloon dilatation of recoarctation was necessary in 3 of your patients, so this problem has not been totally eliminated. However, from the standpoint of growth potential, there certainly is an advantage. How did you determine whether there was a maldistribution of pulmonary blood flow in these patients before the Glenn procedure? Did any of them have scintigraphy, or did you determine angiographically whether there was or was not any left pulmonary artery compression?

Finally, we identified restrictive atrial septal defect as one of the risk factors for early survival for the first-stage procedure. How many of your patients had a severely restrictive atrial septal defect, and what is your current management of these patients?

Dr Poirier. Thank you, Dr del Nido, for your questions.

The small ascending aorta has been identified as a risk factor not only in our group but also in other groups that have used the patch technique and in those that have used the modified technique, meaning without the patch. This finding is not specific to our group.

Yes, the learning curve of a new procedure contributed to this mortality. However, the fact that this technique includes what becomes a small coronary artery might be an intrinsic part of the anatomy that causes this higher mortality. Although we were not able to do a multivariate analysis to identify all the variables that contributed most to the mortality, I think the anatomy played an important part in this mortality.

As for determining the pulmonary artery flow, all of the patients having subsequent procedures underwent angiographic evaluation. At this point, we also measured the pressures, and that is how we determined whether these patients could undergo CPB and a Fontan operation. More important, however, all of these patients had their pulmonary arteries measured in the operating room, even though their pulmonary anatomy seemed favorable and no obstructions were apparent. If the size was inadequate, these patients were treated with an autologous pericardial patch. Thus, the intraoperative investigation was also important.

We thought about the possibility of the atrial septal defect being restrictive, but we did not include that in our analysis of these patients. Actually, we find that a non-critically restrictive atrial septal defect is beneficial, in that it increases the pulmonary vascular resistance in these patients preoperatively, so that they can be stabilized before being brought into the operating room, and allows for good systemic perfusion.

Dr S. Bert Litwin (Milwaukee, Wis). Dr Mee has made many contributions to the surgical treatment of congenital heart disease, not the least of which was popularizing the use of phenoxybenzamine for $\alpha$-blockade in infants undergoing complex operations.

From the Children's Hospital of Wisconsin, Milwaukee, we recently reported at another meeting our results with the Norwood operation in 92 consecutive infants having surgery from January 1992 through December 1999. We divided these into two groups, those seen before July 1996 and those after July 1996. In the latter group, we had added 5 treatment strategies, including new operative techniques, as described by Dr Mee, the use of intraoperative aprotinin and modified ultrafiltration to reduce the inflammatory response to bypass, $\alpha$-blockade with phenoxybenzamine, and postoperative monitoring of myocardial oxygen consumption. The difference in survival was significant, with about a 54\% survival in group I and a $91 \%$ survival in group II. Small aortic size was not a risk factor in our series, although age less than 1 week and absence of the 5 new treatment strategies were.

We also used the strategy of manipulating systemic resistance after the operation while maintaining a normal carbon dioxide tension and ventilatory parameters. Finally, we have found that monitoring of myocardial oxygen consumption is the most sensitive measurement of cardiac performance and oxygen delivery after the Norwood operation.

How did you monitor the degree of $\alpha$-blockade after the operation, and what parameters do you use to either increase or decrease systemic resistance after surgery?

Dr Poirier. Thank you for your questions.

We did not use all the techniques that you have described. However, we did monitor perfusion very closely on the basis of vital signs, skin temperatures, urine output, serial blood gases, and more recently, blood lactate levels. With these we were able to follow our systemic perfusion quite well. However, we did not use your methods. 